\title{
Implicit LES for Supersonic Microramp Vortex Generator: New Discoveries and New Mechanisms
}

\author{
Qin Li and Chaoqun Liu \\ Math Department, University of Texas at Arlington, 411 S. Nedderman Drive, Arlington, TX 76019-0408, USA \\ Correspondence should be addressed to Chaoqun Liu, cliu@exchange.uta.edu
}

Received 6 October 2010; Accepted 12 January 2011

Academic Editor: Guan Yeoh

Copyright () 2011 Q. Li and C. Liu. This is an open access article distributed under the Creative Commons Attribution License, which permits unrestricted use, distribution, and reproduction in any medium, provided the original work is properly cited.

This paper serves as a summary of our recent work on LES for supersonic MVG. An implicitly implemented large eddy simulation (ILES) by using the fifth-order WENO scheme is applied to study the flow around the microramp vortex generator (MVG) at Mach 2.5 and $\operatorname{Re}_{\theta}=1440$. A number of new discoveries on the flow around supersonic MVG have been made including spiral points, surface separation topology, source of the momentum deficit, inflection surface, Kelvin-Helmholtz instability, vortex ring generation, ring-shock interaction, 3D recompression shock structure, and influence of MVG decline angles. Most of the new discoveries, which were made in 2009 , were confirmed by experiment conducted by the UTA experimental team in 2010 . A new 5-pair-vortex-tube model near the MVG is given based on the ILES observation. The vortex ring-shock interaction is found as the new mechanism of the reduction of the separation zone induced by the shock-boundary layer interaction.

\section{Introduction}

It is well known that for the supersonic ramp jet flow, shock boundary layer interaction (SBLI) can significantly degrade the quality of the flow field by triggering largescale separation, causing total pressure loss, making the flow unsteady and distorted, and can even make an engine unable to start. In order to improve the "health" of the boundary layer, a series of new devices named as micro vortex generator is designed for flow control, which is with a height approximately $20-40 \%$ (more or less) of the boundary layer thickness. Because of the robust structure, the specific microramp vortex generator (MVG) becomes more attractive to the inlet designer. Intensive computational and experimental studies have been made on the MVG recently.

Lin indicated [1] that the device like MVG could alleviate the flow distortion in compact ducts to some extent and control boundary layer separation due to the adverse pressure gradients. Similar comments were made in the review by Ashill et al. [2]. The formal and systematic studies about the micro VGs including micro ramp VG can be found in the paper by Anderson et al. [3]. Babinsky et al. [4-7] made a series of experiments on different kinds of mirco VGs and investigated their control effects in details. The mechanism of MVG flow control from his work was described as that a pair of counter-rotating primary streamwise vortices is generated by MVG, which is mainly located within the boundary layer and travel downstream for a considerable distance. Secondary vortices are located underneath the primary ones and even more streamwise vortices could be generated under suitable conditions. Streamwise vortices inside the boundary later will bring low-momentum fluids up from the bottom and high-momentum fluids down to the boundary layer. A striking circular momentum deficit region is observed in the wake behind the MVG. The vortices will keep lifting up slowly, which is thought to be the consequence of the upwash effect of the vortices.

Numerical simulations have been made on MVG for comparative study and further design purposes. Ghosh et al. [8] made detailed computations under the experimental conditions given by Babinsky by using RANS, hybrid RANS/LES, and immersed boundary (IB) techniques. Lee et al. [9, 10] also made computations on the micro VGs problems 


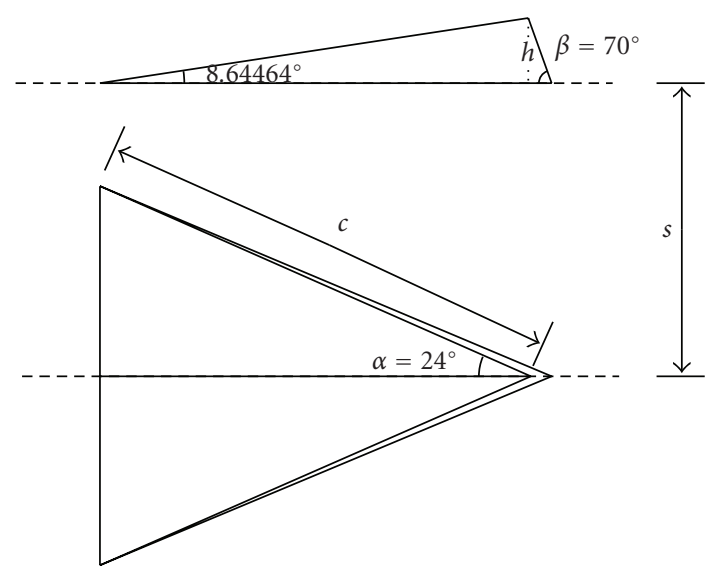

Figure 1: The geometry of MVG.

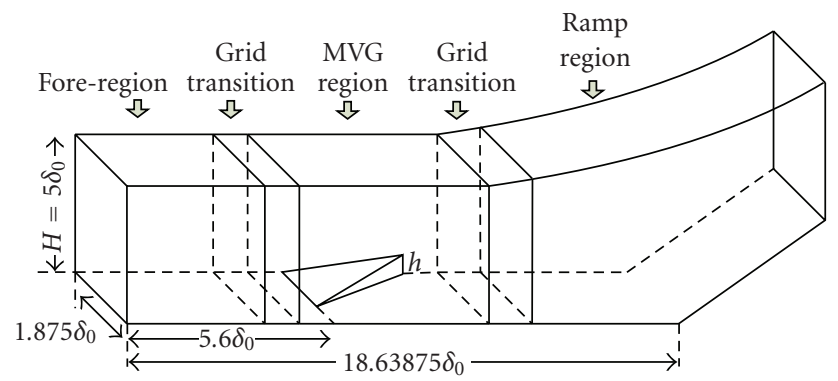

Figure 2: The schematic of the half-grid system of case (1).

by using Monotone Integrated Large Eddy Simulations (MILES). Basic flow structures like momentum deficit and streamwise vortices were reproduced in the computation. Further studies were also conducted on the improvement of the control effect.

Finding physics of MVG for design engineers is definitely needed. RANS, DES, RANS/DES, RANS/LES, and so forth are good engineering tools, but may not be able to reveal the mechanism and get deep understanding of MVG. We need high-order DNS/LES. A powerful tool is the integration of high-order LES and experiment. Recently, an implicit LES for MVG to control the shock-boundary layer interaction around a 24 degree ramp at Mach number of 2.5 and Reynolds number of 1440 has been carried out in 2009 [12]. In this work, several new discoveries have been made and the mechanism of MVG is found quite different from those reported by previous experimental and numerical work in the literature. The new findings include (1) spiral points and flow topology around MVG, (2) new theory of five pairs of vortices near MVG, (3) origin of momentum deficit, (4) inflection points (surface for 3D) and Kelvin-Helmholtz- (K$\mathrm{H}-)$ type instability, (5) vortex ring generation, (6) ringshock interaction and separation reduction, (7) 3D recompressed shock structure, and (8) effects of trailing-edge decline angles.

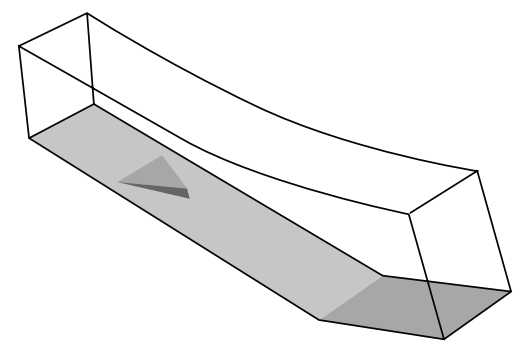

(a) Computational domain

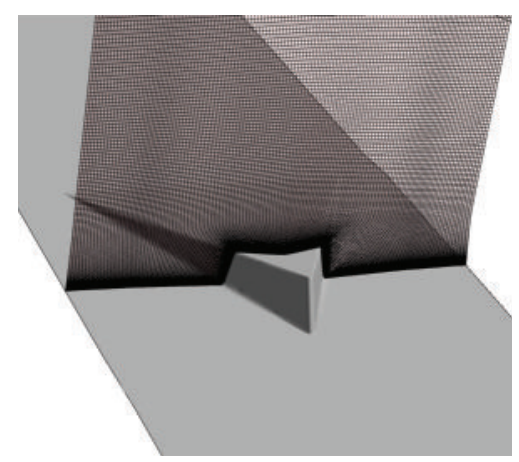

(b) The grid section intersecting the middle of MVG

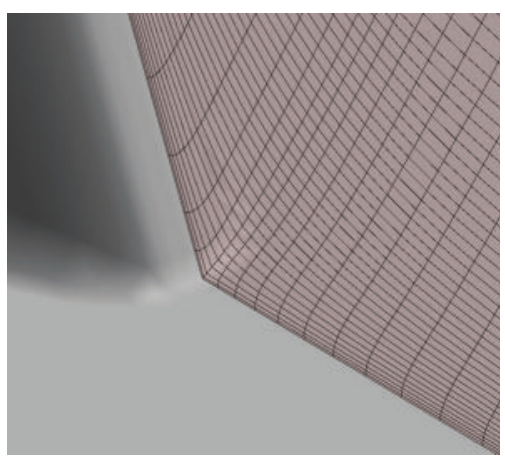

(c) The grids at the corner of MVG

Figure 3: Body-fitted grid system.

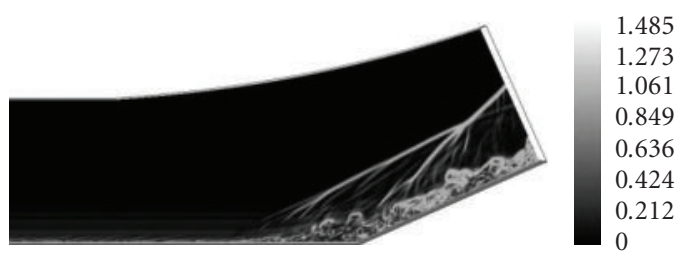

FIgure 4: The digital schlieren at central plane.

Most of the new discoveries were made by October 2009 and were later confirmed by the UTA experimental work in April 2010 [13]. Based on our observation, the mechanism of MVG to reduce the flow separation is really caused by interaction of shock and vortex rings generated by MVG. 


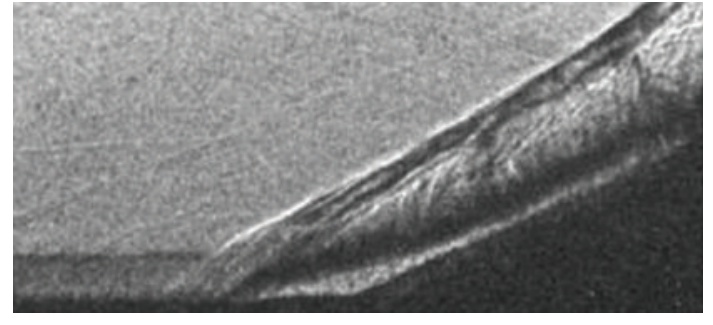

FIgure 5: The experimental schlieren by Loginov et al. [11].

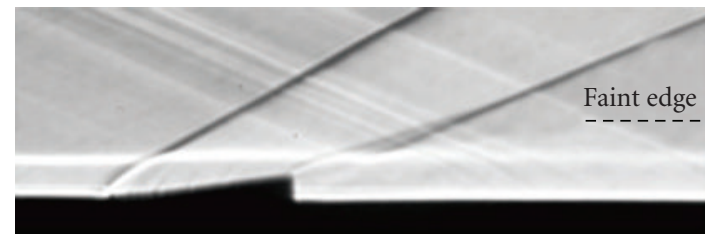

Figure 6: The experimental schlieren by Babinsky et al. [7].
The paper is arranged as following. The numerical methods are discussed in Section 2. In Section 3, all new discoveries are summarized and analyzed and the new mechanism is described. Finally, conclusions are made in Section 4 .

\section{Numerical Methods}

2.1. Governing Equations. The governing equations are the nondimensional Navier-Stokes equations in conservative form as follows:

$$
\frac{\partial Q}{\partial t}+\frac{\partial E}{\partial x}+\frac{\partial F}{\partial y}+\frac{\partial G}{\partial z}=\frac{\partial E_{v}}{\partial x}+\frac{\partial F_{v}}{\partial y}+\frac{\partial G_{v}}{\partial z}
$$

where

$$
\begin{aligned}
& Q=\left[\begin{array}{c}
\rho \\
\rho u \\
\rho v \\
\rho w \\
e
\end{array}\right], \quad E=\left[\begin{array}{c}
\rho u \\
\rho u^{2}+p \\
\rho u v \\
\rho u w \\
(e+p) u
\end{array}\right], \quad F=\left[\begin{array}{c}
\rho v \\
\rho v u \\
\rho v^{2}+p \\
\rho v w \\
(e+p) v
\end{array}\right], \quad G=\left[\begin{array}{c}
\rho w \\
\rho w u \\
\rho w v \\
\rho w^{2}+p \\
(e+p) w
\end{array}\right] \\
& E_{v}=\frac{1}{\operatorname{Re}}\left[\begin{array}{c}
0 \\
\tau_{x x} \\
\tau_{x y} \\
\tau_{x z} \\
u \tau_{x x}+v \tau_{x y}+w \tau_{x z}+q_{x}
\end{array}\right], \quad F_{v}=\frac{1}{\operatorname{Re}}\left[\begin{array}{c}
0 \\
\tau_{y x} \\
\tau_{y y} \\
\tau_{y z} \\
u \tau_{y x}+v \tau_{y y}+w \tau_{y z}+q_{y}
\end{array}\right], \quad G_{v}=\frac{1}{\operatorname{Re}}\left[\begin{array}{c}
0 \\
\tau_{z x} \\
\tau_{z y} \\
\tau_{z z} \\
u \tau_{z x}+v \tau_{z y}+w \tau_{z z}+q z
\end{array}\right] \\
& e=\frac{p}{\gamma-1}+\frac{1}{2} \rho\left(u^{2}+v^{2}+w^{2}\right), \quad q_{x}=\frac{\mu}{(\gamma-1) M_{\infty}^{2} \operatorname{Pr}} \frac{\partial T}{\partial x}, \quad q_{y}=\frac{\mu}{(\gamma-1) M_{\infty}^{2} \operatorname{Pr}} \frac{\partial T}{\partial y}, \\
& q_{z}=\frac{\mu}{(\gamma-1) M_{\infty}^{2} \operatorname{Pr}} \frac{\partial T}{\partial z}, \quad p=\frac{1}{\gamma M_{\infty}^{2}} \rho T, \quad \operatorname{Pr}=0.72 \text {, } \\
& \tau=\mu\left[\begin{array}{ccc}
\frac{4}{3} \frac{\partial u}{\partial x}-\frac{2}{3}\left(\frac{\partial v}{\partial y}+\frac{\partial w}{\partial z}\right) & \frac{\partial u}{\partial y}+\frac{\partial v}{\partial x} & \frac{\partial u}{\partial z}+\frac{\partial w}{\partial x} \\
\frac{\partial u}{\partial y}+\frac{\partial v}{\partial x} & \frac{4}{3} \frac{\partial v}{\partial y}-\frac{2}{3}\left(\frac{\partial w}{\partial z}+\frac{\partial u}{\partial x}\right) & \frac{\partial v}{\partial z}+\frac{\partial w}{\partial y} \\
\frac{\partial u}{\partial z}+\frac{\partial w}{\partial x} & \frac{\partial v}{\partial z}+\frac{\partial w}{\partial y} & \frac{4}{3} \frac{\partial w}{\partial z}-\frac{2}{3}\left(\frac{\partial u}{\partial x}+\frac{\partial v}{\partial y}\right)
\end{array}\right] .
\end{aligned}
$$

The viscous coefficient is given by Sutherland's equation:

$$
\mu=T^{3 / 2} \frac{1+C}{T+C}, \quad C=\frac{110.4}{T_{\infty}} .
$$

The nondimensional variables are defined as follows:

$$
\begin{array}{ccc}
x=\frac{\tilde{x}}{L}, & y=\frac{\tilde{y}}{L}, & z=\frac{\tilde{z}}{L}, \\
u=\frac{\tilde{u}}{U_{\infty}}, & v=\frac{\tilde{v}}{U_{\infty}}, & w=\frac{\tilde{w}}{U_{\infty}},
\end{array}
$$

$$
\begin{gathered}
T=\frac{\tilde{T}}{T_{\infty}}, \quad \mu=\frac{\tilde{\mu}}{\mu_{\infty}}, \quad k=\frac{\tilde{k}}{k_{\infty}}, \\
\rho=\frac{\tilde{\rho}}{\rho_{\infty}}, \quad p=\frac{\tilde{p}}{\rho_{\infty} U_{\infty}^{2}}, \quad e=\frac{\tilde{e}}{\rho_{\infty} U_{\infty}^{2}},
\end{gathered}
$$

where the variables with “ ” are the dimensional counterparts. 


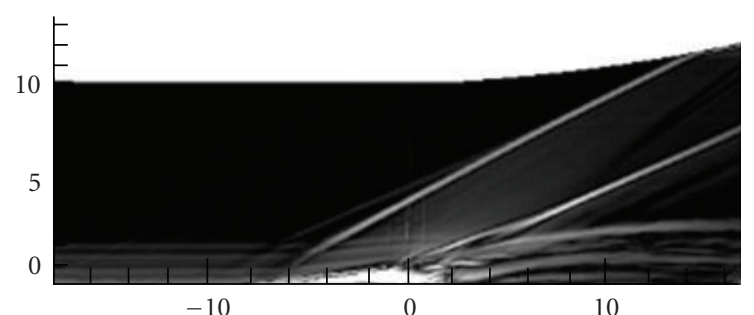

$-10$

10

Figure 7: The numerical schlieren at central plane.

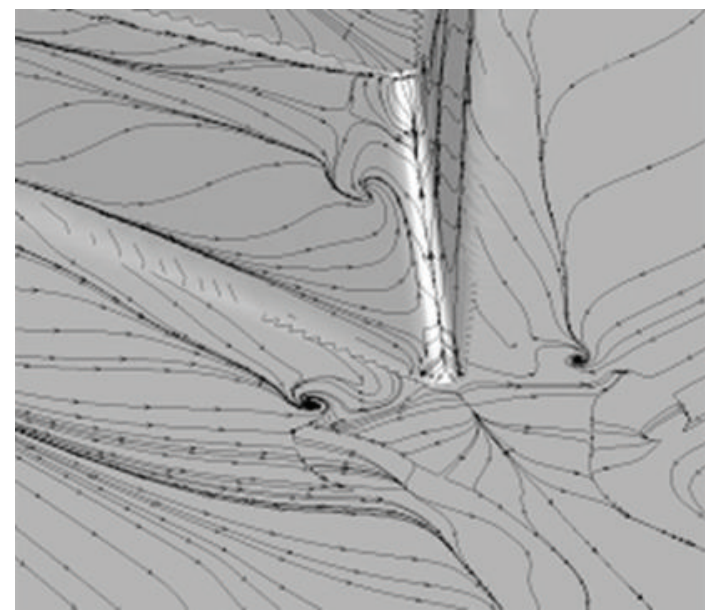

FIGURE 8: Spiral point pairs by ILES in 2009.

Considering the following grid transformation,

$$
\begin{aligned}
\xi & =\xi(x, y, z), \\
\eta & =\eta(x, y, z), \\
\zeta & =\zeta(x, y, z),
\end{aligned}
$$

the Navier-Stokes equations can be transformed to the system using generalized coordinates:

$$
\frac{\partial \hat{Q}}{\partial \tau}+\frac{\partial \hat{E}}{\partial \xi}+\frac{\partial \hat{F}}{\partial \eta}+\frac{\partial \hat{G}}{\partial \zeta}=\frac{\partial \hat{E}_{v}}{\partial \xi}+\frac{\partial \hat{F}_{v}}{\partial \eta}+\frac{\partial \hat{G}_{v}}{\partial \zeta}
$$

where $\hat{Q}=J^{-1} Q$ and

$$
\begin{aligned}
\hat{E} & =J^{-1}\left(\xi_{x} E+\xi_{y} F+\xi_{z} G\right), \\
\hat{F} & =J^{-1}\left(\eta_{x} E+\eta_{y} F+\eta_{z} G\right), \\
\hat{G} & =J^{-1}\left(\zeta_{x} E+\zeta_{y} F+\zeta_{z} G\right), \\
\hat{E}_{v} & =J^{-1}\left(\xi_{x} E_{v}+\xi_{y} F_{v}+\xi_{z} G_{v}\right), \\
\hat{F}_{v} & =J^{-1}\left(\eta_{x} E_{v}+\eta_{y} F_{v}+\eta_{z} G_{v}\right), \\
\hat{G}_{v} & =J^{-1}\left(\zeta_{x} E_{v}+\zeta_{y} F_{v}+\zeta_{z} G_{v}\right),
\end{aligned}
$$

$J^{-1}, \xi_{x}$, and so forth, are grid metrics, and $J^{-1}=\operatorname{det}(\partial(x$, $y, z) / \partial(\xi, \eta, \zeta)), \xi_{x}=J\left(y_{\eta} z_{\zeta}-z_{\eta} y_{\zeta}\right)$, and so forth.

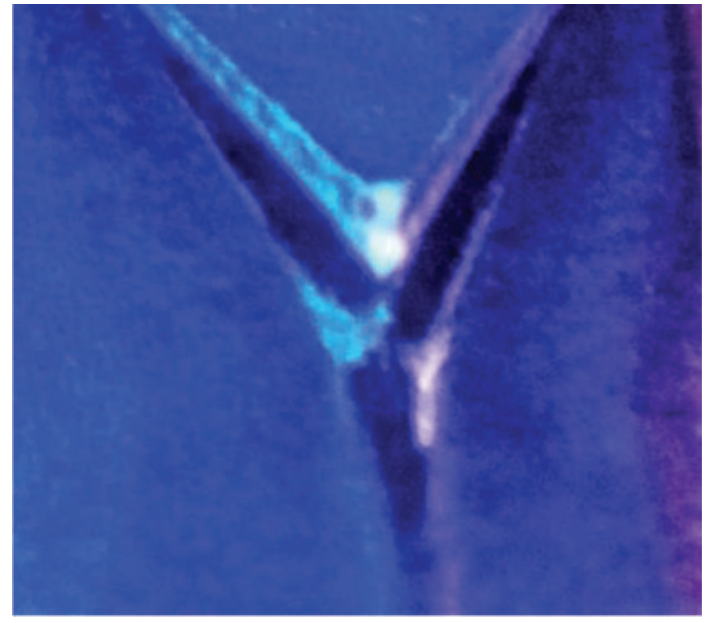

FIGURE 9: Oil accumulation blocks by UTA experiment in 2010.

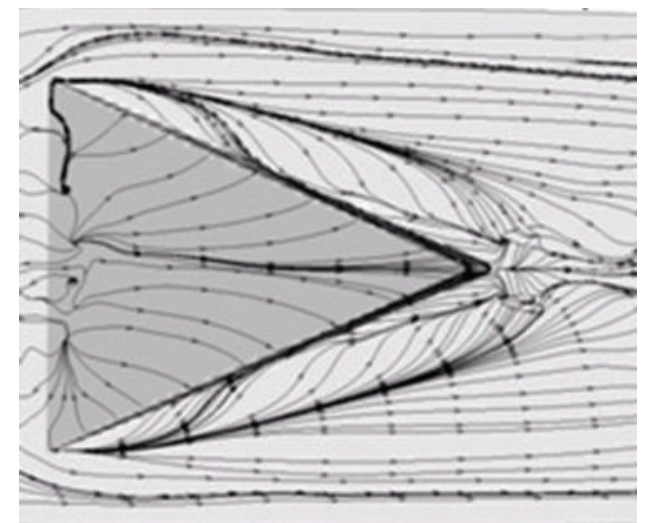

(a)

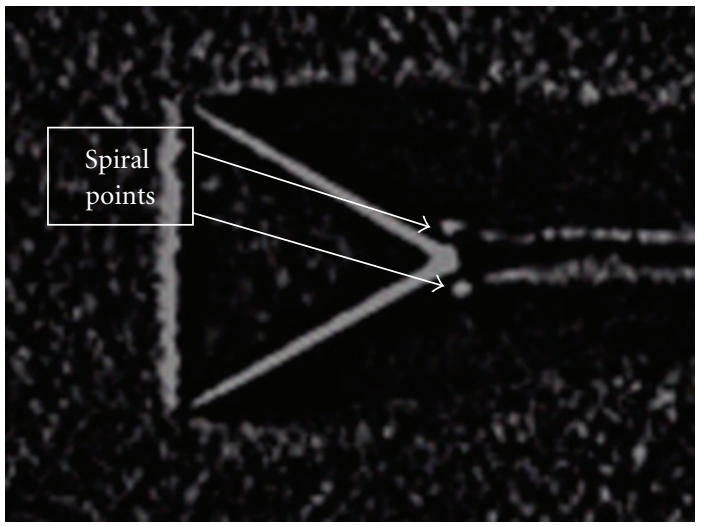

(b)

FIGURE 10: Comparison of ILES with UTA experiment.

\subsection{Finite Difference Schemes and Boundary Conditions}

1. The Fifth-Order WENO Scheme for the Convective Terms [14]. In order to decrease the dissipation of the scheme, the less dissipative Steger-Warming flux splitting method is used in the computation, but not the commonly used dissipative Lax-Friedrich splitting method. 


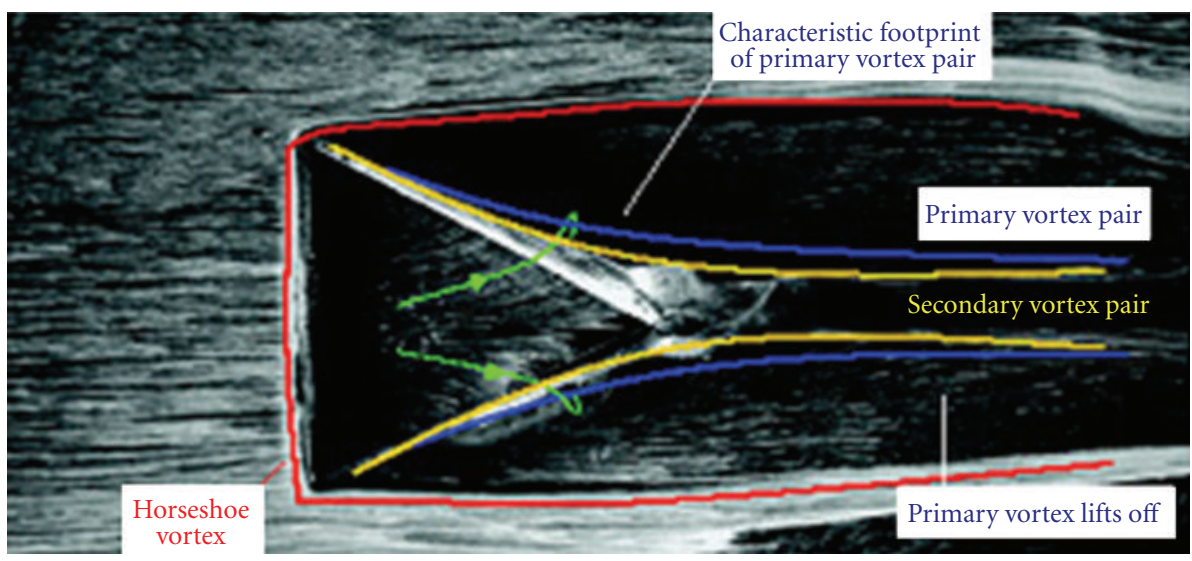

FIGURE 11: Traditional vortex structure around MVG.

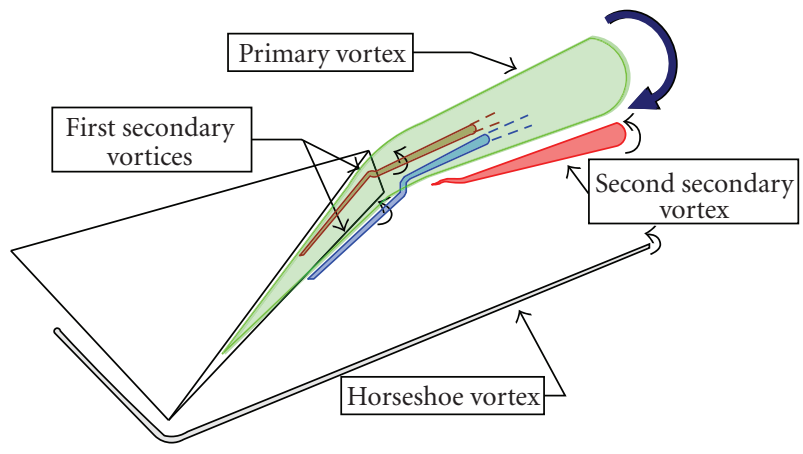

Figure 12: New 5-pair-vortex-tube model near MVG.

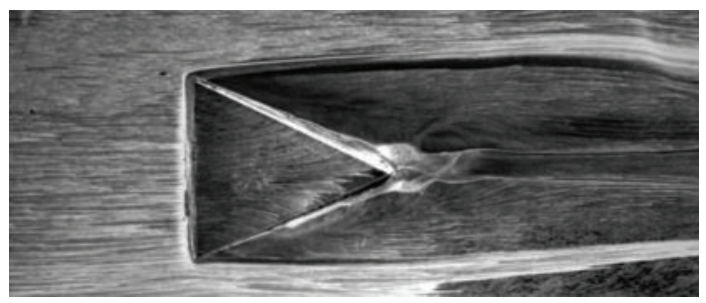

FIgUre 13: The surface oil flow by Babinsky7.

2. The Difference Scheme for the Viscous Terms. Considering the conservative form of the governing equations, the traditional fourth-order central scheme is used twice to compute the second-order derivatives for viscous terms.

3. The Time Schems. The basic methodology for the temporal terms in the Navier-Stokes equations adopts the explicit third-order TVD-type Runge-Kutta scheme:

$$
\begin{gathered}
u^{(1)}=u^{n}+\Delta t L\left(u^{n}\right), \\
u^{(2)}=\frac{3}{4} u^{n}+\frac{1}{4} u^{(1)}+\frac{1}{4} \Delta t L\left(u^{(1)}\right), \\
u^{n+1}=\frac{1}{3} u^{n}+\frac{2}{3} u^{(2)}+\frac{2}{3} \Delta t L\left(u^{(2)}\right) .
\end{gathered}
$$

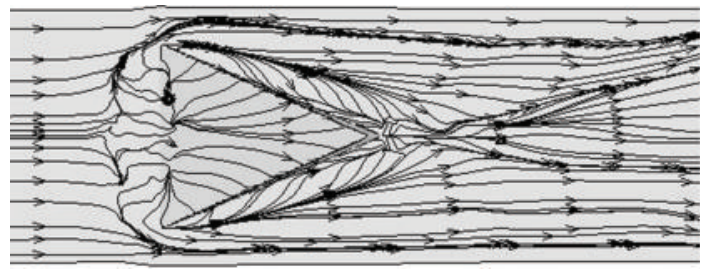

FIgURE 14: The surface limiting streamlines by computation.

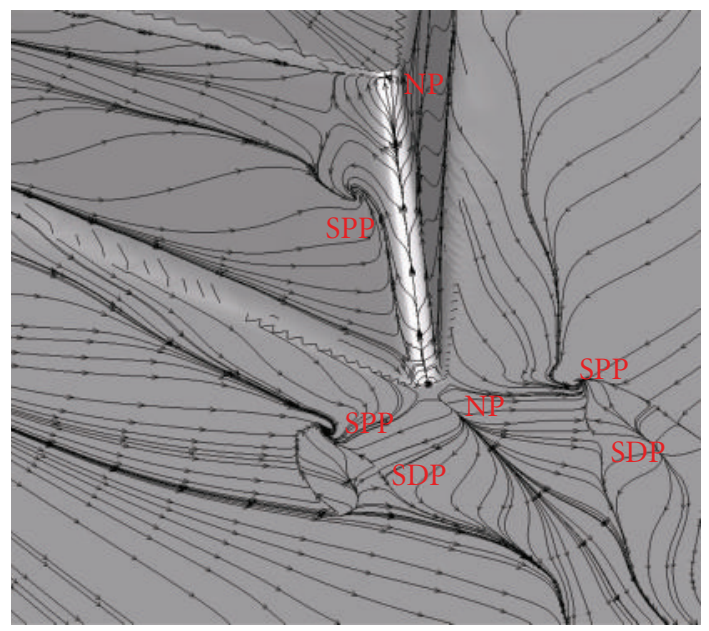

Figure 15: The separation pattern near the end of MVG.

4. Boundary Conditions. The adiabatic, zero-gradient of pressure and nonslipping conditions are used for the wall as

$$
\frac{\partial T}{\partial n}=0, \quad \frac{\partial p}{\partial n}=0, \quad \vec{U}=0 .
$$

To enforce the free stream condition, fixed-value boundary condition with the free parameters is used on the upper boundary. The boundary conditions at the front and back boundary surface in the spanwise direction are given as the mirror-symmetry condition. The outflow boundary conditions are specified as a kind of characteristic-based condition, which can handle the outgoing flow without 


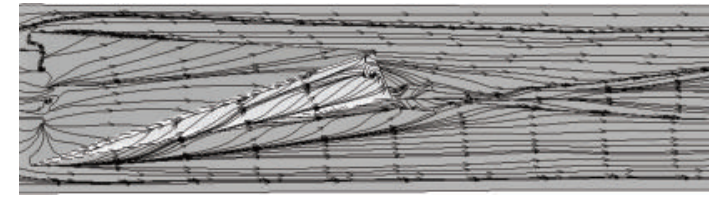

(a) SSP on the side of MVG

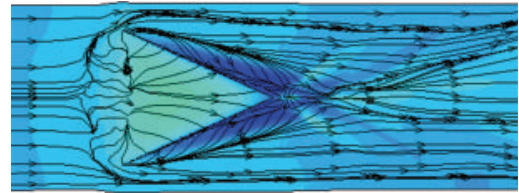

(b) SSP backgrounded using the pressure contour

FIgURE 16: The various views of surface separation pattern (SSP).

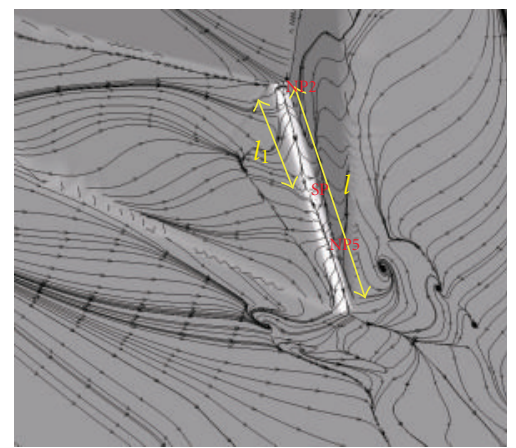

(a) Computation: $l_{1} / l=0.57$

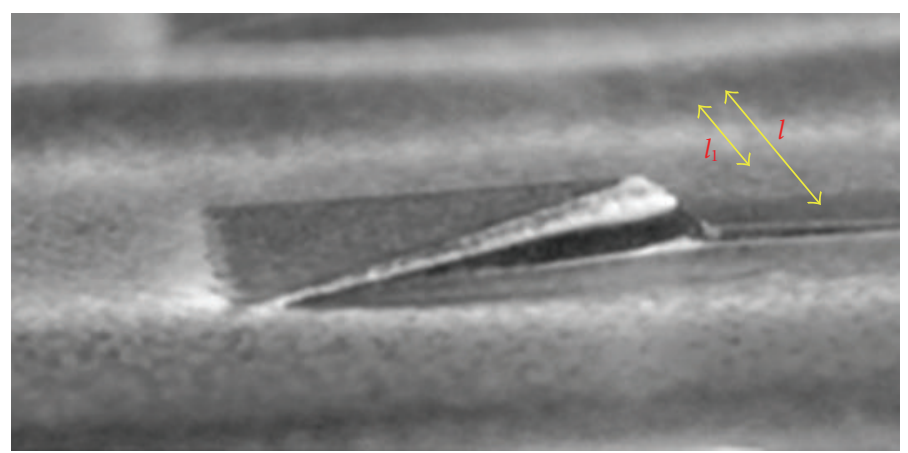

(b) Experiment: $l_{1} / l=0.545$

FIGURE 17: Comparison of ILES and experiment in surface flow topology.

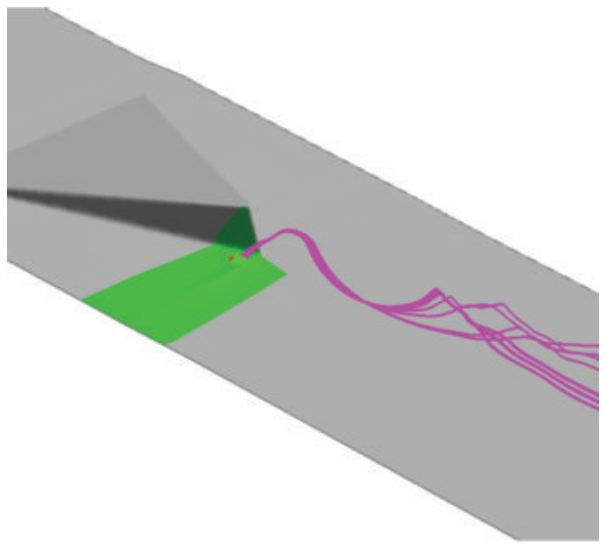

(a) Secondary vortex lifts up

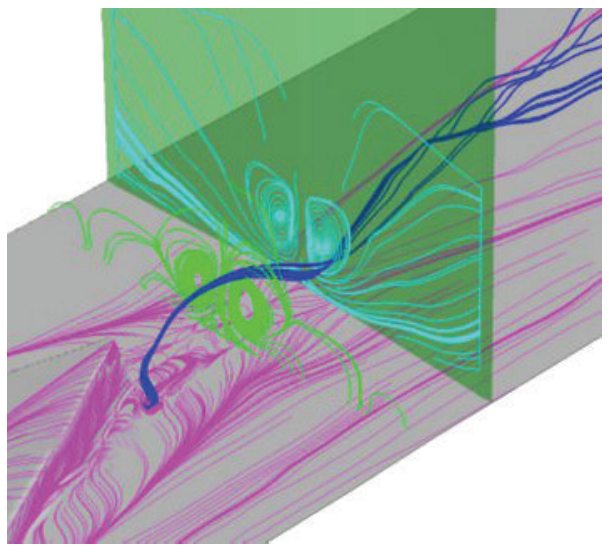

(b) Moving downstream around the primary

FIgURE 18: Secondary vortex tubes lift up from the bottom plate.

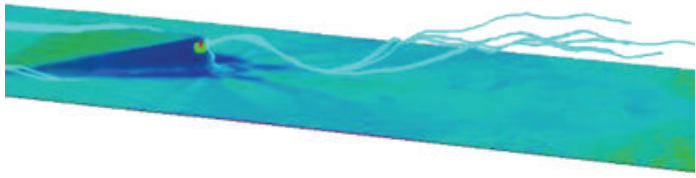

(a) Secondary vortex lifts up

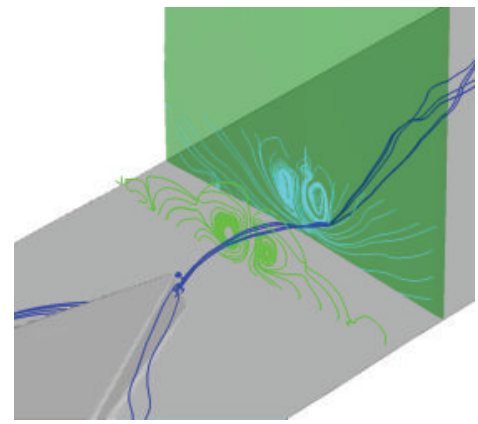

(b) Moving downstream around the primary

FIgURE 19: Secondary vortex tubes lift up from MVG sides. 


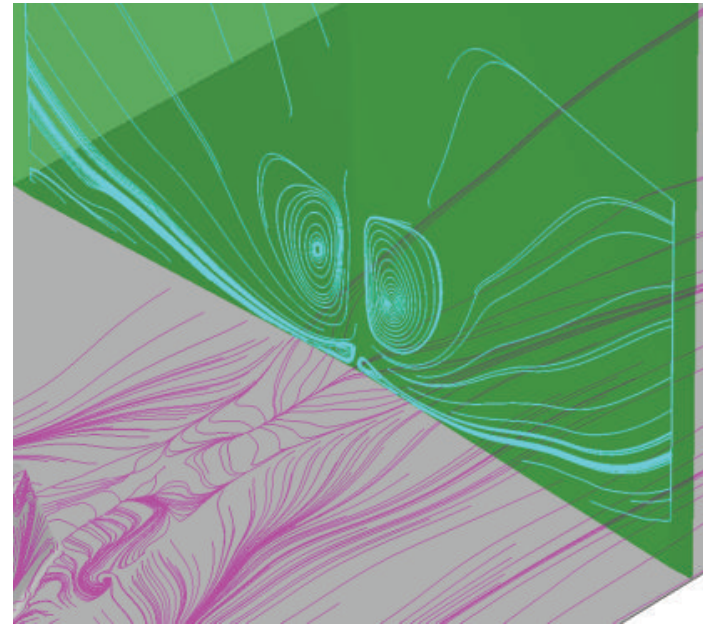

Figure 20: New secondary vortex tubes induced by the primary.

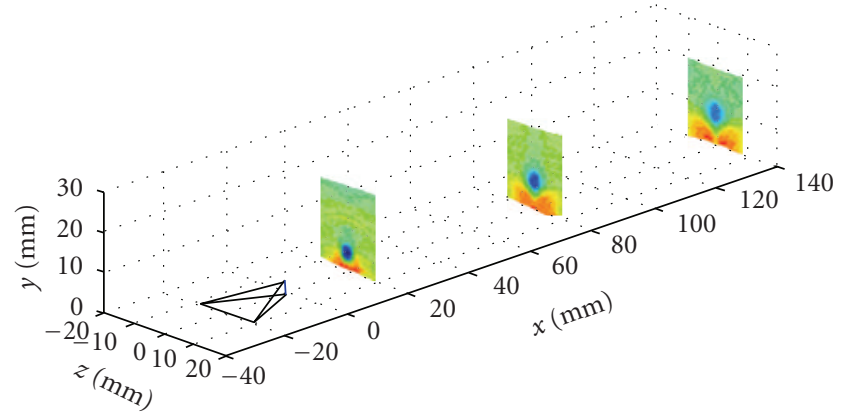

FIGURE 21: Momentum deficit shown in different streamwise directions.

reflection. The inflow conditions are generated using the following steps.

(a) A turbulent mean profile is obtained from DNS by Liu and Chen [15] for the streamwise velocity ( $w$ velocity) and the distribution is scaled using the local displacement thickness and free stream velocity.

(b) Random fluctuations are added on the primitive variables, that is, $u, v, w, p, \rho$. The disturbance has the form: $\varepsilon_{\text {distb }} e^{-\left(y-y_{w}\right)^{2} / \Delta y_{\text {distb }}} \times($ random -0.5$) / 2$, where the subscript "distb" means the disturbance, "random" is the random function with the value between $0 \sim 1, \varepsilon_{\text {distb }}$ equals to 0.1 , and $\Delta y_{\text {distb }}$ equals to $2 / 3 \delta_{0}$.

Such inflow conditions are, of course, not the fully developed turbulent flow, but we can consider it as a weakly disturbed inflow while propagating downstream.

5. Body-Fitted Grid Generation. The geometry of MVG is shown in Figure 1. In order to alleviate the difficulty to grid generation caused by original vertical trailing-edge, a modification is made by declining the edge to $70^{\circ}$. The other geometric parameters in the figure are the same as those given by Holden and Babinsky [5], that is, $c=7.2 h, \alpha=24^{\circ}$, and $s=7.5 h$, where $h$ is the height of MVG and $s$ is the distance between the center lines of two adjacent MVGs. So the distance from the center line to the spanwise boundary of the computation domain is 3.75h.According to experiments by Holden and Babinsky [5], the ratio $h / \delta_{0}$ of the models has the range from $0.3 \sim 1$. The appropriate distance from the trailing-edge to the control area is around $19 \sim 56 h$ or $8 \sim$ $19 \delta_{0}$. So in this study, the height of MVG $h$ is assumed to be $\delta_{0} / 2$ and the horizontal distance from the apex of MVG to the ramp corner is set to be $19.5 \mathrm{~h}$ or $9.75 \delta_{0}$. The distance from the end of the ramp to the apex is $32.2896 h$. The distance from the starting point of the domain to the apex of MVG is $17.7775 h$. The height of the domain is from $10 h$ to $15 h$ and the width of the half domain is $3.75 h$. The geometric relation of the half of the domain can be seen in Figure 2, where the symmetric plane is the centre plane.The grid number for the whole system is $n_{\text {spanwise }} \times n_{\text {normal }} \times n_{\text {streamwise }}=128 \times 192$ $\times 1600$. We try to make the grids smooth and orthogonal as much as possible (Figure 3).

\subsection{Code Validation}

1. Supersonic Ramp. Figure 4 gives the instantaneous numerical schlieren image of the central plane, which uses the value of $|\nabla \rho|$. For qualitative comparison, an experimental picture of ramp flow at $M=2.9$ with large Reynolds number is given in Figure 5 obtained from the experiment by Loginov et al. [11]. From both pictures, we can observe that the separation shock wave has a declining angle which is a little lager but nearly same to the ramp angle and is almost aligned with the reflection shock (which is more obvious in the picture of the experiment); complex compressive waves are generated underneath the shock by vortices in the boundary layer.

2. Supersonic MVG. Based on the schlieren picture of the experiment, Babinsky et al. [7] presented the structure of the wave system, that is, the first reflection shock, the expansion wave system, and the recompression shock, as shown in Figure 6. The first shock will bend within the incoming boundary layer due to "the change in Mach number", and "a second shock wave then turns the flow back to horizontal". A subtle oblique " $\lambda$ " structure is observed at the foot of the recompression shock from the picture. The numerical schlieren picture of the time-averaged flow field at the central plane is presented in Figure 7. From the figure, the two shock waves are well described by the computation. The bending of the main shock to the wall can be distinguished; the oblique " $\lambda$ " structure can be found on the foot of the second shock as well. Table 1 gives a comparison between numerical and experimental results. The measured shock angles from Babinsky's experiment are $26.869^{\circ}$ for the first shock and $21.93^{\circ}$ for the recompression shock. In contrast, the computational values are $26.988^{\circ}$ for the first shock, and $24.656^{\circ}$ for the recompression shock. Considering the declining angle of the trailing-edge is $70^{\circ}$, not the $90^{\circ}$ as in the experiment, the computational results show a reasonable agreement with experiment and of a high degree of resolution. 


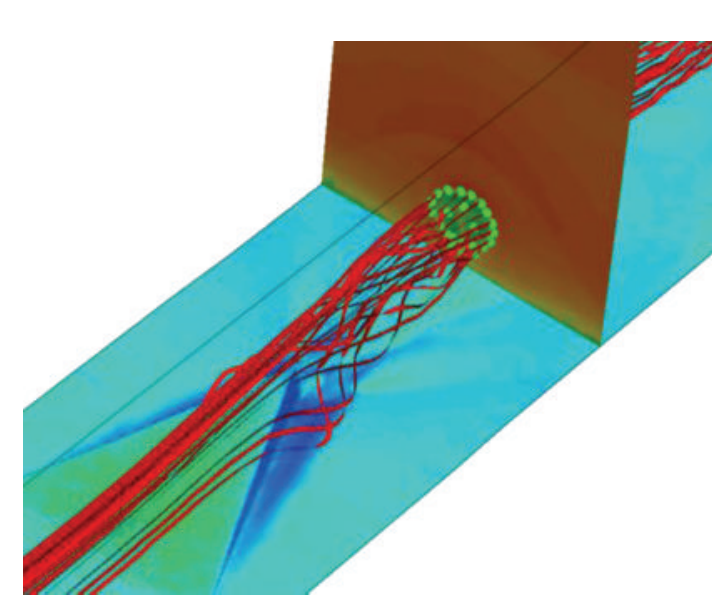

(a) Stream track for momentum deficit origin

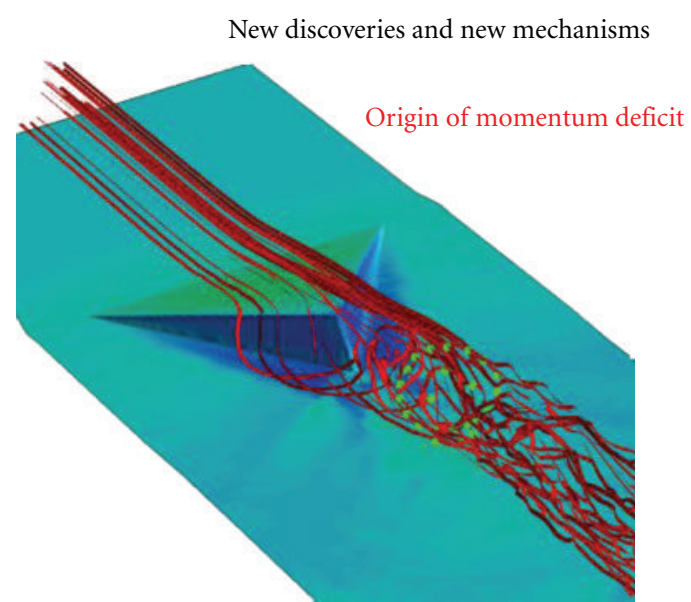

(b) Locally enlarged stream tracks

FIgURE 22: Origin of the momentum deficit comes from the upper surface flow of MVG entrained by the primary vortex tubes.

TABLE 1: Comparison between IlES and experiment in shock angles.

\begin{tabular}{lcc}
\hline & 1 st shock & 2nd shock \\
\hline Experiment & $26.869^{\circ}$ & $21.93^{\circ}$ \\
Computation & $26.988^{\circ}$ & $24.656^{\circ}$ \\
\hline
\end{tabular}

\section{New Discoveries}

3.1. Discovery I: Spiral Points and Vortex Structure around $M V G$. A pair of spiral points on the bottom plate and another pair on the sides of MVG (Figure 8) have been found by ILES. These spiral points indicate the rising from the wall surface of the secondary vortex pairs. These new findings have been confirmed by UTA oil flow experiment (Figure 9), which shows accumulation blocks of paint. A further comparison is given in Figure 10.

3.2. Discovery II: New Model of Five Pairs of Vortices near $M V G$. The traditional vortex structure around MVG is depicted in Figure 11 by Babinsky et al. [7], that is, a pair of counter-rotating primary streamwise vortices is generated by MVG, which is mainly located within the boundary layer and travel downstream for a considerable distance. Secondary vortices are located underneath the primary ones and even more streamwsie vortices could be generated under suitable conditions. However, a new model of 5 pairs of vortex tubes near MVG is given by us in Figure 12. The main difference between the two models is about the secondary vortex structures. There are two pairs of rising secondary vortex tubes corresponding to two pairs of spiral points. After the two pairs of secondary vortex tubes rising, a new pair of the secondary tubes is induced by the primary vortex tubes. Of course, there is additional one pair of horseshoe vortices. Recently, in April 2010, the experimentalists in the University of Texas at Arlington (UT Arlington) took a video from the top view to record the process of the MVG oil flow. A distinct pair of oil accumulating points was found in the video (see the experimental snapshot in Figures 9 and 10).
3.3. Discovery III: Surface Separation Topology. For comparison, the pictures of the surface oil flow from experiment and limiting streamlines from the computation are given in Figures 13 and 14. The topology of the two figures is essentially the same. The separation lines of the horseshoe vortex, the secondary separation lines beside the MVG, and the ones after the MVG are clearly described by computation (Figures 15 and 16). Such lines are the most obvious traces found in experiment due to the deposit of the oil. Figure 17 provides a comparison of computation and experiment in surface topology. The black area in experiment shows no oil flow from MVG top surface, which is corresponding to flow separation in computation. The agreement between computation and experiment is well obtained.

The original secondary vortex tubes induced by the primary vortex tubes on the bottom plate and MVG sides were lifted up from the spiral points and further move downstream around the primary (Figures 18 and 19). After that, a pair of new secondary vortex tubes is induced by the primary vortex tubes (Figure 20).

3.4. Discovery IV: Origin of Momentum Deficit. The momentum deficit is a unique phenomenon which was first observed in the experiment by Ford and Babinsky [6], and later confirmed by the computation of Ghosh et al. [8] and Lee et al. [10], as shown in Figure 21, using the averaged streamwise velocity at different sections. About the mechanism of the deficit, Babinsky thought it is the wake of MVG, and Lee only mentioned "the two vortical tubes merge together to create a larger tube with two counter-rotating vortices inside". What is the relation of the deficit to the flow structure? What does the low-speed flow come from? The existing explanations did not provide a clear mechanism about the formation of the deficit.

In order to investigate the origins of the deficit, a heuristic analysis is made by investigating specific streamlines that are defined by certain cross-section of the momentum deficit. First, a cross-section after the MVG is selected as a reference 


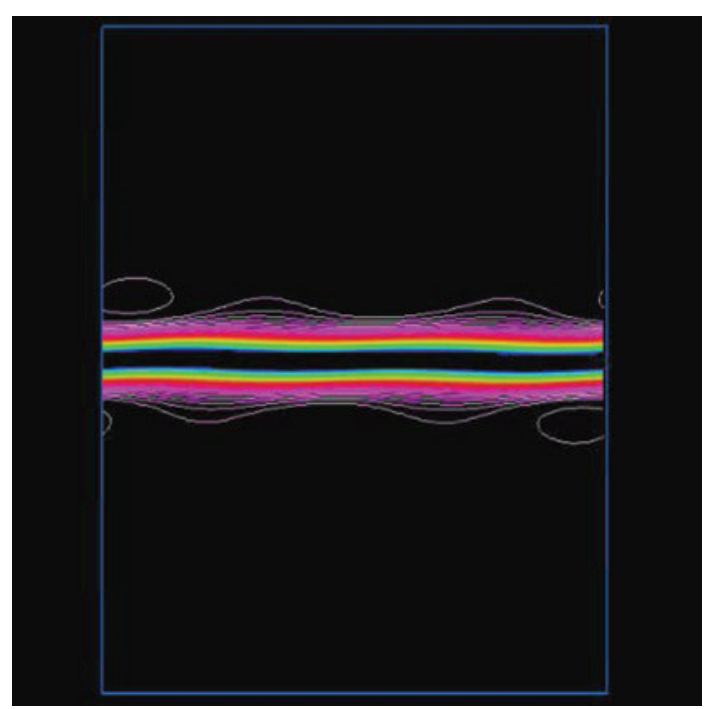

(a)

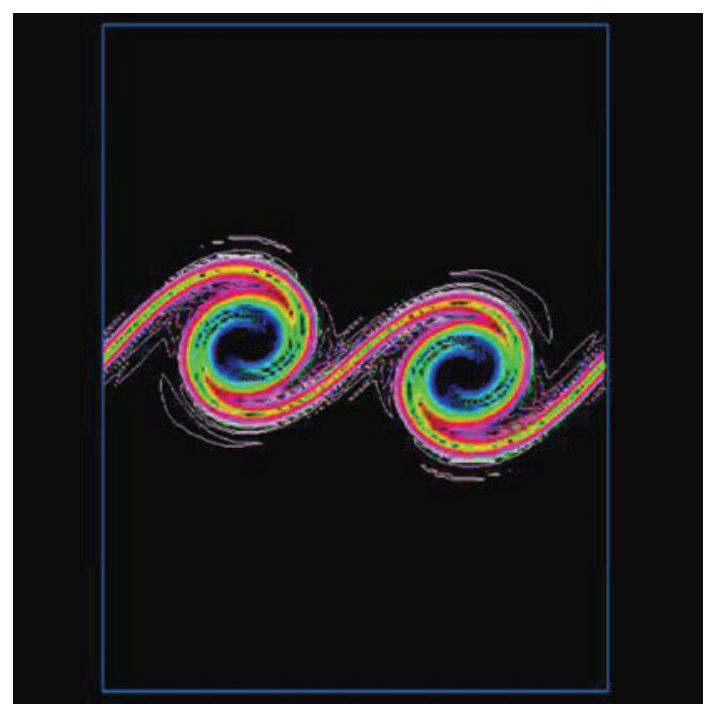

(c)

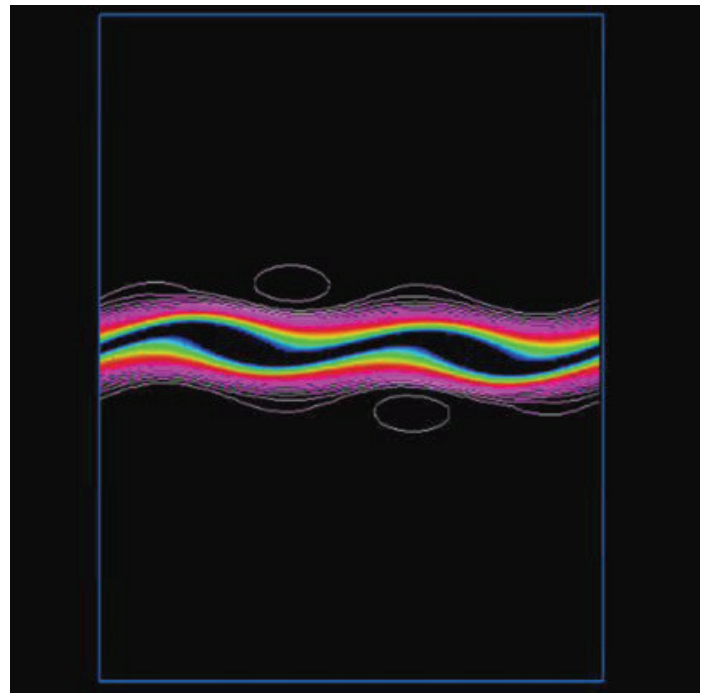

(b)

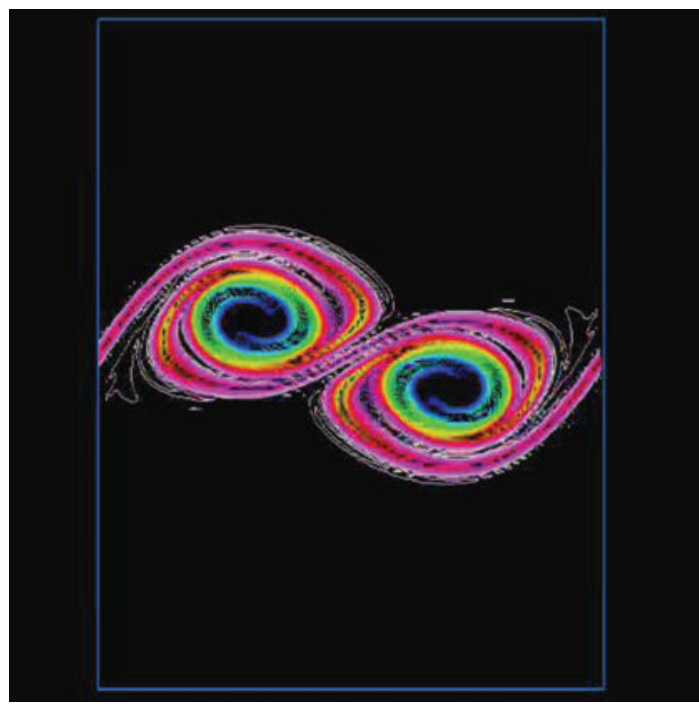

(d)

Figure 23: 2D Kelvin-Helmoholzs instability and vortex ring formation.

plane as shown in Figure 22, and the momentum deficit on the plane is shown by using the contour of the instantaneous streamwise velocity, which appears in a green circular region. Next, we put some seeds around the boundary of the deficit and within the deficit area. Then we draw the 3D streamlines starting from the seeds in both backward and forward directions. Because the seeds surround or locate within the deficit, the distribution of the backward streamlines qualitatively reflects the origins of the deficit. It can be seen from Figure 22 that (a) all backward streamlines rotate around the primary streamwise vortex, which indicates that the formation of the deficit is caused by primary vortices; (b) all backwards streamlines come from the upper surface of the MVG, which indicates that the main source of the deficit is the shedding of the upstream low-speed boundary layer, but is not the boundary layer flow around the MVG or after the MVG.

\subsection{Discovery V: Inflection Surface, K-H Instability, and Vortex Ring Generation by $M V G$}

3.5.1. 2D Kelvin-Helmoholz Instability. 2D instability caused by shear layer with inflow disturbances and formation of pairing vortex rings was obtained by our previous calculation (Figure 23). It is usually called Kelvin-Helmholtz instability which is described as inviscid instability.

3.5.2. Inflection Surface in $3 D$ Flow behind MVG. In order to explore the mechanism of the vortex ring generation, the distributions of averaged streamwise velocity are given in Figure 24 along the normal grid lines at the center plane. The streamwise positions of the lines are $L_{\text {from apex }} / h \approx 3.3$, 6.7, 10 , and 11 , where $L_{\text {from apex }}$ is the streamwise distance measured from the apex of MVG. The dip of the lines 


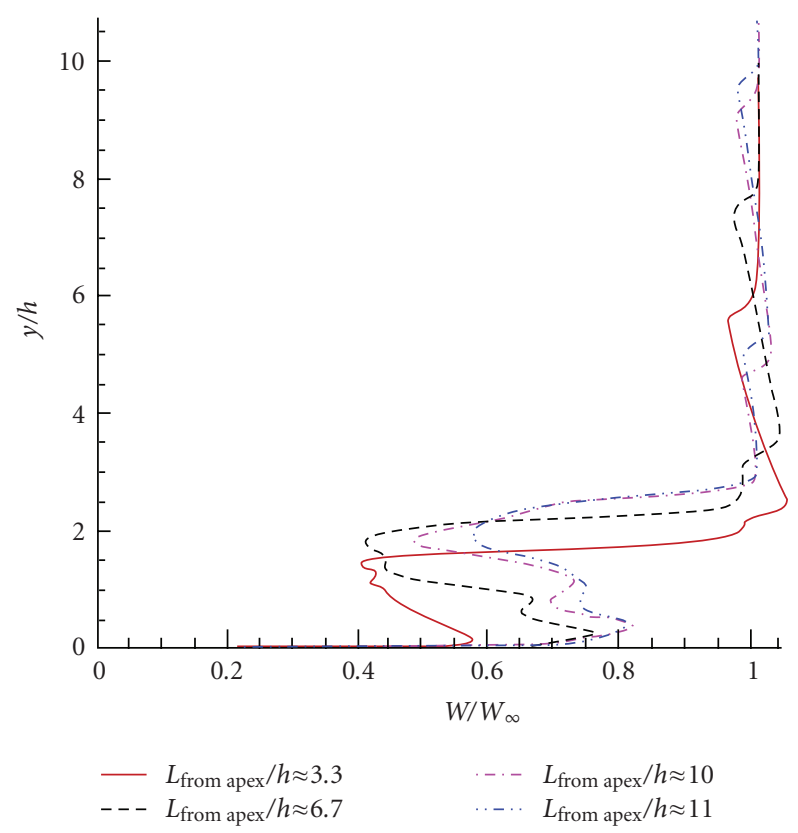

Figure 24: Averaged streamwise velocity at different sections.

corresponds to the momentum deficit. From the results, it can be clearly seen that there are at least two highshear layers in the central plane: one is located at the upper edge of the dip and the other is located at the lower edge. Within the shear layer, there is at least one inflection point. In order to demonstrate the existence of the inflection points, the second-order derivative $\partial_{2} w / \partial y_{2}$ ( $w$ is the streamwise velocity and $y$ is the normal direction) is calculated along the lines, and the result of the line at $L_{\text {from apex }} / h \approx 6.7$ is plotted in Figure 25 as an example. The existence and correspondence of the inflection points at the upper and lower shear layers is illustrated by two dashed lines intersecting the distribution of the streamwise velocity and its second-order derivative.

3.5.3. Vortex Ring Generation. Based on the above analyses, it can be concluded that the existence of the inflection points (surface in 3D) in the shear layer causes the flow instability and generates vortex rollers by K-H instability in a cylindrical coordinate system. Therefore, the mechanism of the vortex ring generation should be $\mathrm{K}-\mathrm{H}$ instability. The lost of the stability of the shear layer will result in the roll-up of the vortex, which appears in ringlike structure in a $3 \mathrm{D}$ view (Figure 26). In the Figure 26(b), $\lambda_{2}$ is a certain eigenvalue of the stress tensor, and its iso-surface is usually used to describe the vortex surface. The intensity of the upper shear layer appears to be stronger than that of the lower shear layer (Figure 26).

In Figure 27, another qualitative checking about the shear layer and $\mathrm{K}-\mathrm{H}$ instability is made by using the instantaneous flow field. In the figure, the background at the central plane and the spanwise plane is colored by the value of the streamwise velocity, so that the green regions in two planes represent the momentum deficit. In the central plane,

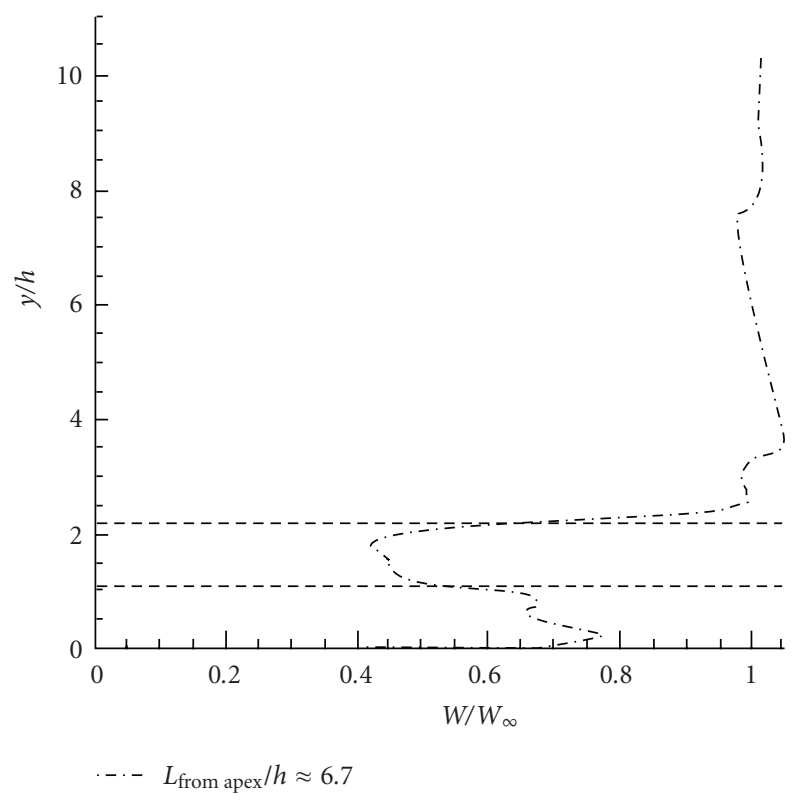

(a) Averaged streamwise velocity at $L_{\text {from apex }} / h \approx 6.7$

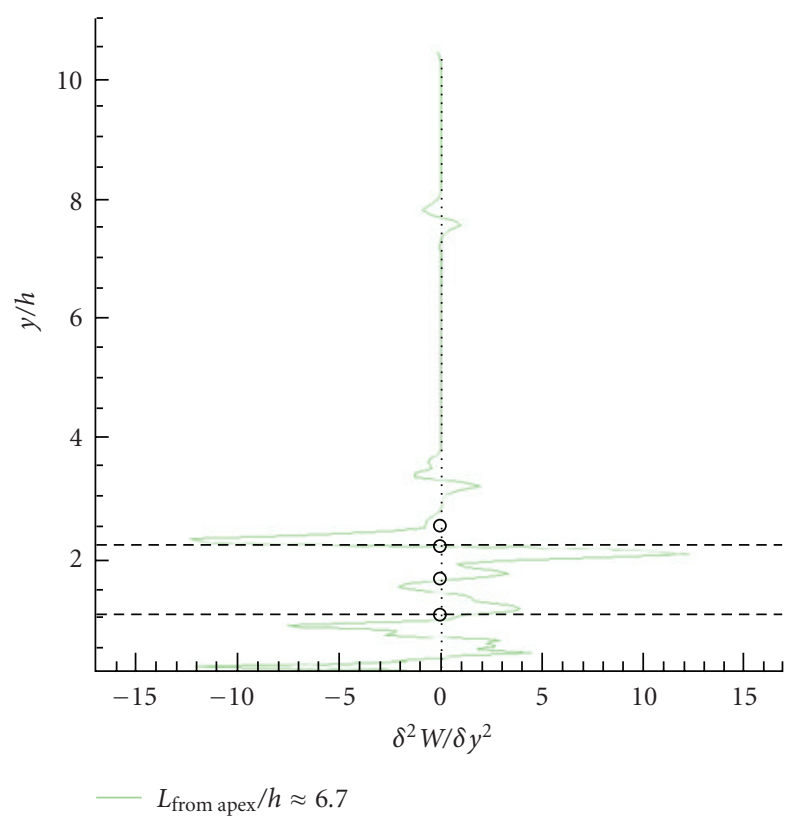

(b) $\partial^{2} u / \partial y^{2}=0$

FIGURE 25: Inflection points (surface for 3D).

the pressure contours are superimposed on the background cloud-map. The figure shows that the blue circle structures, which indicate the core of the ringlike vortices cut by the plane, are located on the boundary of the deficit circle. Such positions are exactly the same place where the high-shear layer exists.

In Figure 28, we give the instantaneous numerical schlieren picture at the central plane. From the figure, we can see many vortex rings appear in the circular shapes; after being told the prediction of the vortex rings, the same experimentalists in UT Arlington tried some techniques 


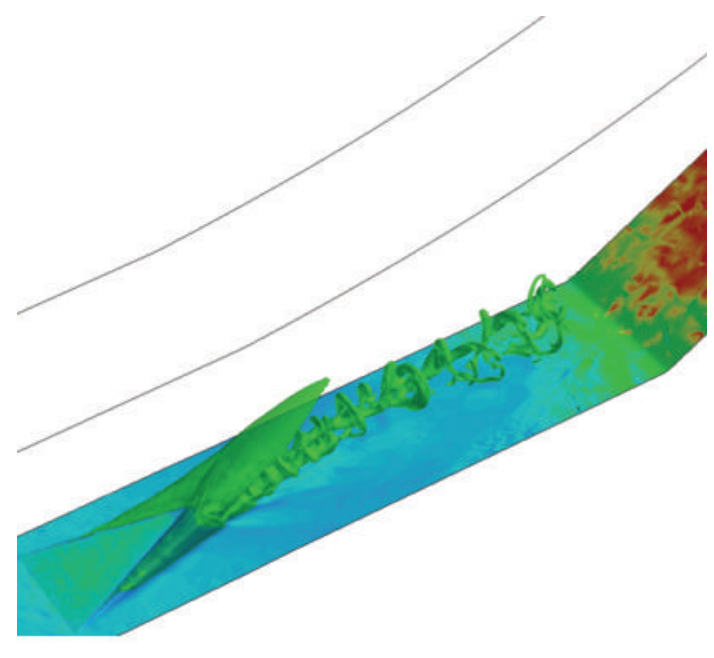

(a) Iso-surface of pressure

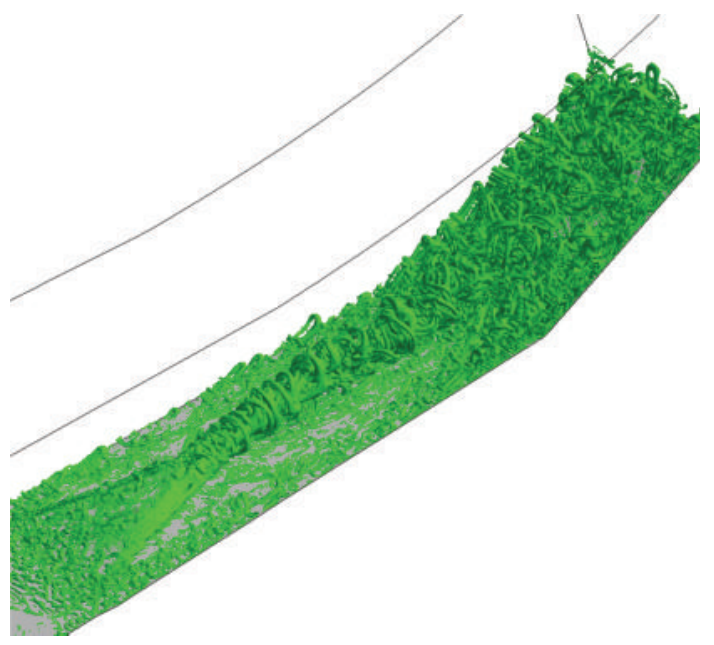

(b) Iso-surface of $\lambda_{2}$

FIGURE 26: Vortex ring generation by MVG due to K-H instability.

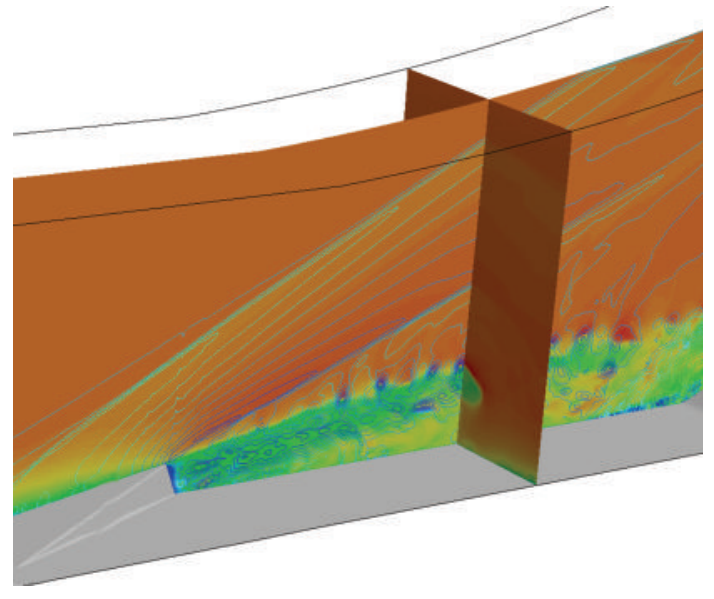

FIGURE 27: The instantaneous pressure and streamwise velocity contour on different cross-sections.

to validate the discovery. They used techniques of the particle image velocimetry (PIV) and the acetone vapor screen visualization to track the movement of the flow, and specifically the flash of a laser sheet is used to provide the light exposure at the time level of micro seconds. In Figure 29, a typical image at the center plane is presented taken by using PIV and the acetone vapor [13]. It is clearly demonstrated that a chain of vortex rings exist in the flow field after the MVG! And these structures qualitatively resemble those in Figures 27 and 28.

\subsection{Discovery VI: Reduction of Separation Zone by Ring-Shock Interaction}

3.6.1. New Mechanism of Separation Zone Reduction by MVG. Traditional concept for MVG is that the MVG produces

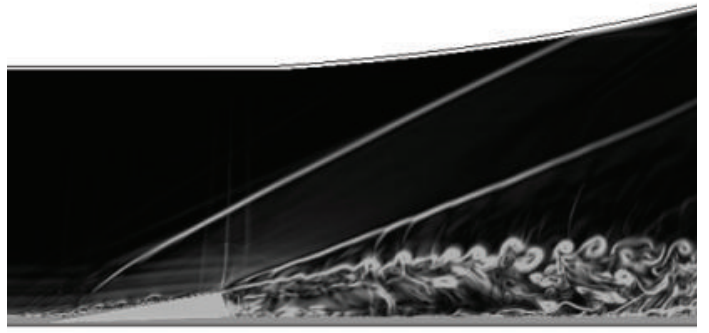

Figure 28: The numerical schlieren at the center plane.

streamwise vortices inside the boundary later, which will bring low-momentum fluid up from the bottom and highmomentum fluid down to the boundary layer and then the flow separation induced by shock will be reduced due to the higher shear. However, our LES just gives a quite different mechanism which can be described as MVG generates vortex rings and vortex rings destroy the shocks and reduce the boundary layer separation bubble size, which was induced by the separation bubble and reflection shocks.

Figure 30 shows a graphic about the shock structure around a supersonic ramp, which includes a separation bubble shock and a reflection shock. The boundary layer separation is caused by the strong adverse pressure gradients induced by the shock. Many vortex rings are generated by MVG and convect downstream toward the ramp. The ring first destroys the separation bubble shock and the separation bubble looks broken (Figure 31). The vortex rings are very stable and continue to move downstream to destroy the reflection shock as shown in Figure 32. After many rings continuously move toward the reflection shock, the shock tail 


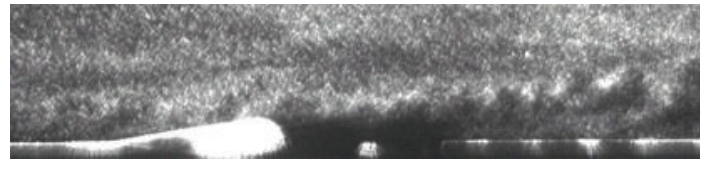

(a) Using PIV

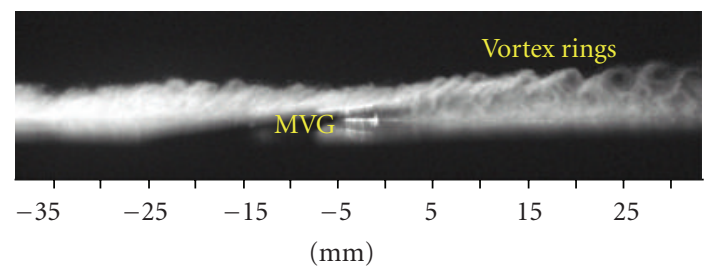

(b) Using the acetone vapor

Figure 29: The laser-sheet flash image at the center plane.

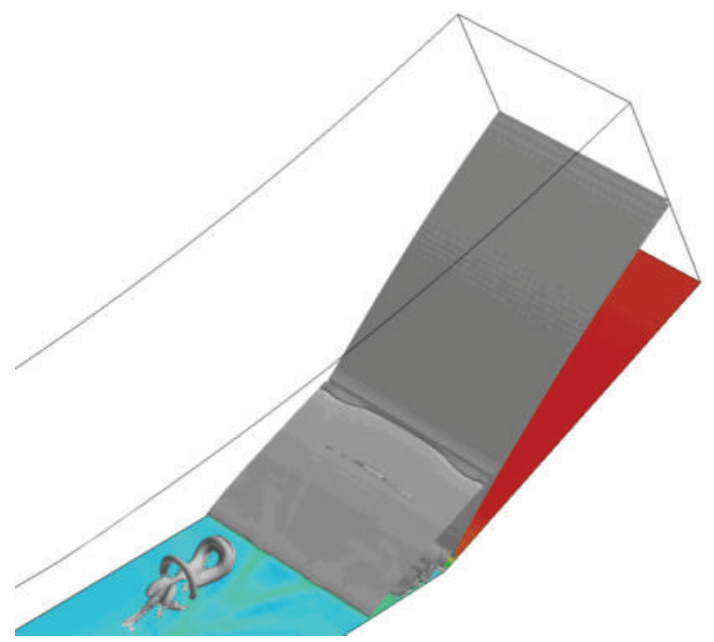

FIGURE 30: Shock structure around ramp.

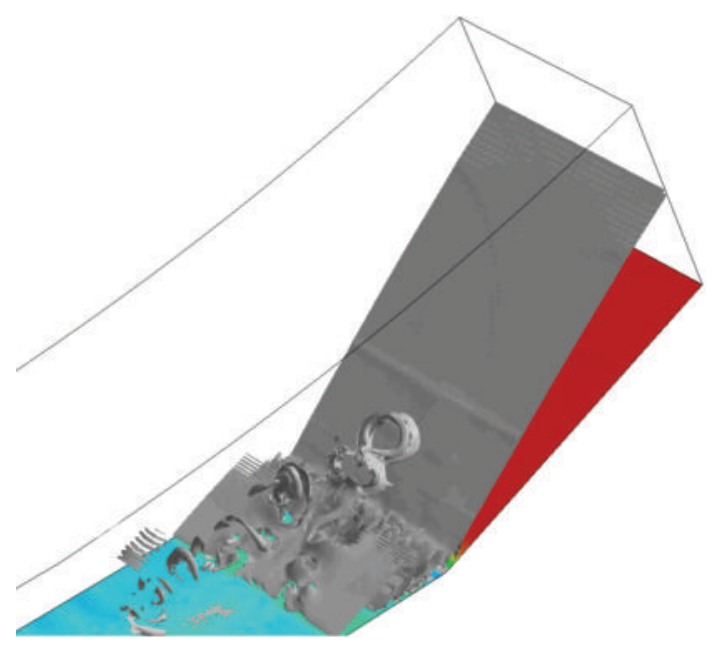

FIGURE 31: Ring-separation bubble shock interaction.

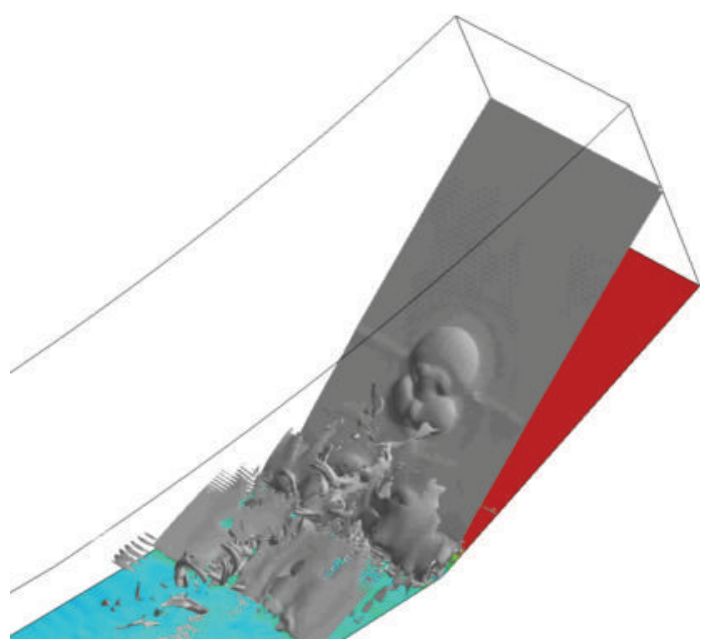

FIGURE 32: Ring-reflection shock interaction.

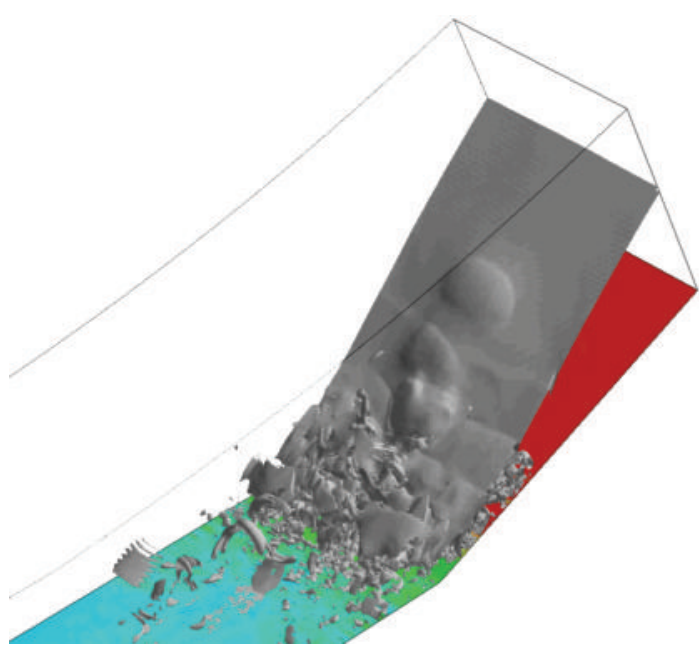

FIGURE 33: Separation reduction by vortex rings.

disappeared and the separation area is substantially reduced (Figure 33).

3.6.2. Shock-Vortex Ring Interaction. The mechanism why the vortex ring can destroy the shock is believed as that the vortex ring has a low pressure line inside the ring which could destroy the shock. On the other hand, the ring has a high-pressure area outside the ring and near the center of ring, which will change the shock location. The fast rotation of the ring could make the shock surface deformed like a bump.

3.6.3. Stable Structure of the Vortex Ring. The shock is pretty strong and the pressure gradients are pretty large. We use both digital schlieren (Figure 34) and $\lambda_{2}$ techniques (Figure 35) for 3D visualization and 2D visualization on a central plane in the spanwise direction. The vortex rings are found very stable and keep their forms unchanged after 


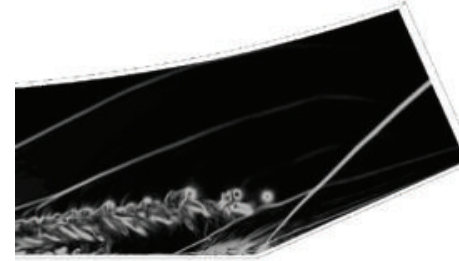

(a) Before the separation shock

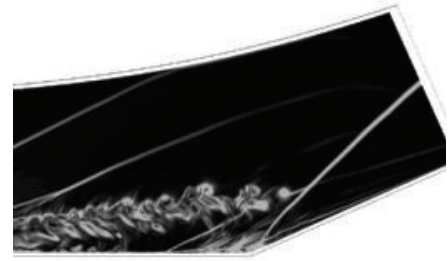

(b) Past the separation shock

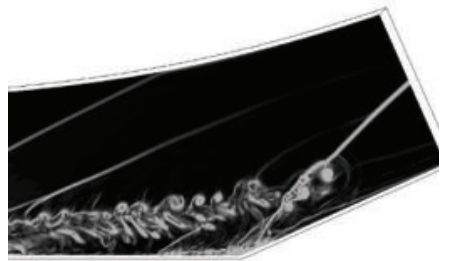

(c) Past the ramp shock

FIGURE 34: Vortex ring-shock interaction at the central plane (numerical schlieren).

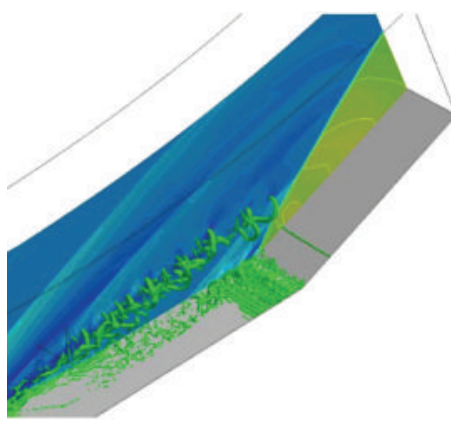

(a) Before the shock

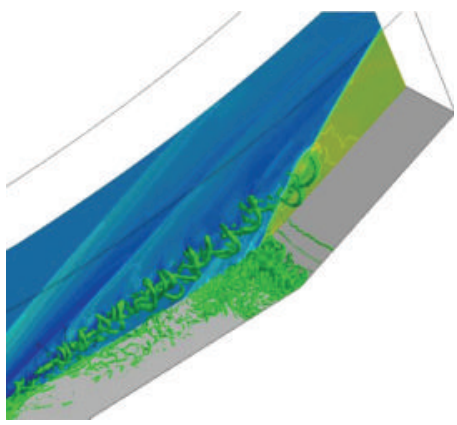

(b) Hit the shock

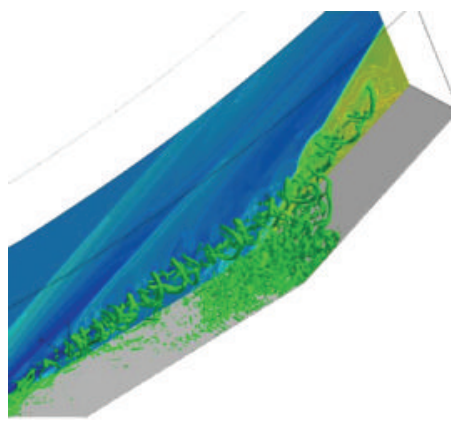

(c) Past the shock

FIGURE 35: Vortex ring-shock interaction at the central plane ( $\lambda_{2}$ techniques).

passing the weak separation bubble shock and strong ramp shock.

\subsection{Discovery VII: Three-Dimensional Recompressed Shock} Structure. In Figure 36, the spatial structures of the wave system are given by instantaneous cross-section pressure contours. From the first section in Figure 36(a), the wave system is found to be the main reflection shock above the MVG and the expansion wave emanating from the edge of the MVG. The remaining sections show an arclike structure of the recompression shock wave, which is located behind the first reflection shock. The size of the arc keeps growing while moving downstream. The curve shape of the recompression shocks implies that the function of the recompression shock wave is to make the expansion flow satisfy the "virtual" boundary condition created by the streamwise vortices, at least at the initial stage of the formation of the shock wave. Detailed investigations show that the head and feet of the arc-like shock are separated from each other in the initial stage (see Figure 36(b)). They begin to connect and form a complete arc curve at a certain downstream location, as shown in Figure 36(c).

3.8. Discovery VIII: Effects of Trailing-Edge Decline Angles. Comparative studies are made on the MVG flow at $M=2.5$ and $\operatorname{Re}_{\theta}=1440$ while the MVGs are of different declining angle, that is, $\beta=70^{\circ}$ and $\beta=45^{\circ}$. The numerical study finds (see Figure 37 ) the following

(1) The basic structures are similar in two cases, like the wave system, separation pattern, momentum deficit, and the vortex rings. The effect of declining angle does not bring about the structural difference, while differences exist in the details of the flow.

(2) The smaller declining angle at $\beta=45^{\circ}$ makes the vortices closer to the wall, which include the initial streamwise vortices and the layer vortex rings. This should be more favorable to flow control. Nevertheless, the quantitative analyses on the time-averaged data at a downstream do not exhibit a significant improvement about the quality of the boundary layer.

\section{Conclusions}

The following new discoveries and new mechanisms have been made by implicit LES since 2009. Most of these new findings were confirmed later by UTA experiment in 2010.

(1) Detailed flow structures (topology) around MVG and spiral points are found. Then the new five pairs of vortex tubes model around MVG are given.

(2) Origin of the momentum deficit is found, not only caused by stream wise vortices through mushroom, but also, mainly, viscosity of MVG and wall surface.

(3) Inflection points (surface in 3D) inside the deficit area are found.

(4) Kelvin-Helmholtz-type instability is caused by the momentum deficit.

(5) Vortex rings are generated by K-H-type instability after MVG.

(6) Shock-ring interaction is found. 


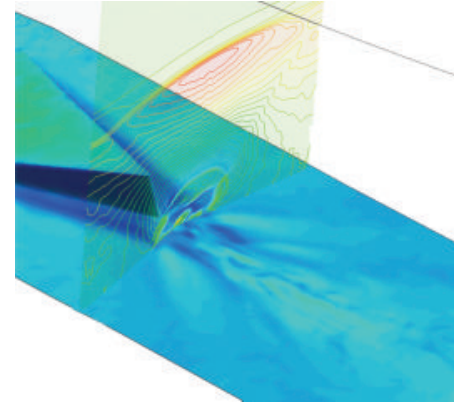

(a) Section 1

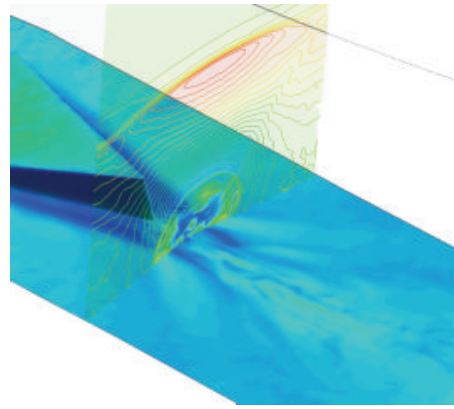

(b) Section 2

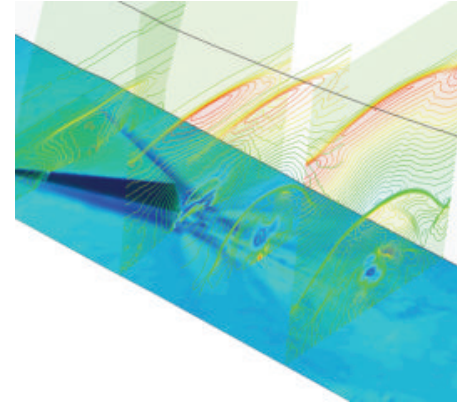

(c) Overview

FIGURE 36: The 3D structure of the recompression shock.

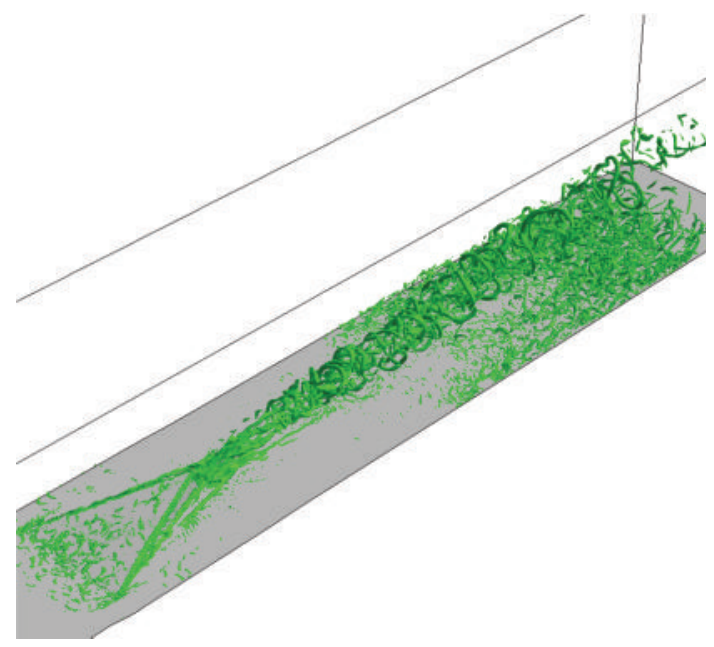

(a) $\beta=70^{\circ}$

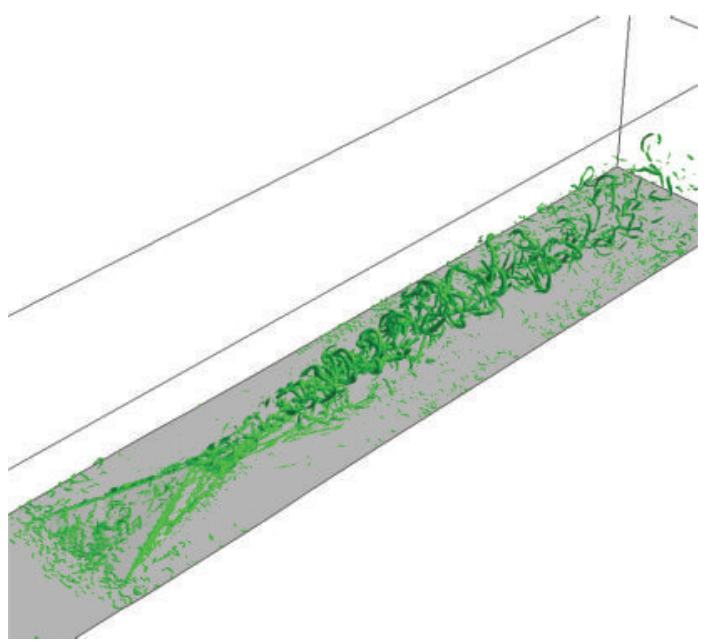

(b) $\beta=45^{\circ}$

FIGURE 37: Comparison of the vortex structure with different decline angles of MVG trailing edge.

(7) Mechanism of MVG for separation reduction is due to the ring shock interaction, not only mixing as traditional explanation on VG or MVG.

(8) Ring is the only form of vortex inside the fluid field and never breaks down.

(9) Structure of recompressed shock is found.

(10) Influence of the MVG trailing edge decline angles is studied.

All these new findings may be very important to regular VG, subsonic flow, flow control, flow transition, and turbulence studies.

\section{Nomenclature}

M: Mach number

$\mathrm{Re}_{\theta}$ : Reynolds number based on momentum thickness

c: Micro ramp vortex generator side length

$h$ : MVG height $\alpha: \quad$ MVG half angle

$\beta$ : $\quad$ MVG declining angle of the trailing edge

$\delta$ : $\quad$ Incompressible boundary-layer nominal thickness

$\delta^{*}: \quad$ Incompressible boundary-layer displacement thickness

$\theta$ : Incompressible boundary-layer momentum thickness

$H_{i}$ : Incompressible boundary-layer shape factor $\delta^{*} / \theta$

$x, y, z$ : Spanwise, normal, and streamwise coordinate axes

$u, v, w$ : Spanwise, normal, and streamwise velocities

$p_{0}: \quad$ Pitot pressure

$C_{\text {Ptot }_{\mathrm{rc}}}$ : Pitot pressure recovery coefficient

Pr: $\quad$ Prandt number

RANS: Reynolds-averaged Navier-Stokes

LES: Large eddy simulation

DNS: Direct numerical simulation

WENO: Weighted essentially nonoscillatory scheme

TVB: Total variation bounded 
SBLI: Shock wave boundary layer interaction VG: Vortex generator

MVG: Microramp VG.

Subscript

$w$ : Wall

$\infty$ : Free stream

0 : Representing the location at the inlet if without special explanation.

\section{Acknowledgments}

This work is supported by AFOSR Grant FA9550-08-1-0201 supervised by Dr. John Schmisseur. The authors are grateful to Texas Advantage Computing Center (TACC) for providing computation hours. The authors thank Dr. Frank Lu for providing some experimental snapshots.

\section{References}

[1] J. C. Lin, "Review of research on low-profile vortex generators to control boundary-layer separation," Progress in Aerospace Sciences, vol. 38, no. 4-5, pp. 389-420, 2002.

[2] P. R. Ashill, J. L. Fulker, and K. C. Hackett, "A review of recent developments in flow control," Aeronautical Journal, vol. 109, no. 1095, pp. 205-232, 2005.

[3] B. H. Anderson, J. Tinapple, and L. Surber, "Optimal control of shock wave turbulent boundary layer interactions using micro-array actuation," in Proceedings of the 3rd AIAA Flow Control Conference, pp. 880-893, San Francisco, Calif, USA, June 2006, AIAA paper 2006-3197.

[4] H. A. Holden and H. Babinsky, "Vortex generators near shock/ boundary layer interactions," in Proceedings of the 42nd AIAA Aerospace Sciences Meeting and Exhibit, pp. 9975-9983, Reno, Nev, USA, January 2004, AIAA paper 2004-1242.

[5] H. Holden and H. Babinsky, "Effect of microvortex generators on separated normal shock/boundary layer interactions," Journal of Aircraft, vol. 44, no. 1, pp. 170-174, 2007.

[6] C. W. P. Ford and H. Babinsky, "Micro-ramp control for oblique shock wave / boundary layer interactions," in Proceedings of the 37th AIAA Fluid Dynamics Conference, pp. 972-985, Reno, Nev, USA, June 2007, AIAA paper 2007-4115.

[7] H. Babinsky, Y. Li, and C. W. P. Ford, "Microramp control of supersonic oblique shock-wave/boundary-layer interactions," AIAA Journal, vol. 47, no. 3, pp. 668-675, 2009.

[8] S. Ghosh, J. I. Choi, and J. R. Edwards, "Numerical simulations of effects of micro vortex generators using immersedboundary methods," AIAA Journal, vol. 48, no. 1, pp. 92-103, 2010.

[9] S. Lee and E. Loth, "Supersonic boundary-layer interactions with various micro-vortex generator geometries," in Proceedings of the 39th AIAA Fluid Dynamics Conference, June 2009, AIAA paper 2009-3712.

[10] S. Lee, M. K. Goettke, E. Loth, J. Tinapple, and J. Benek, "Microramps upstream of an oblique-shock/boundary-layer interaction," AIAA Journal, vol. 48, no. 1, pp. 104-118, 2010.

[11] M. S. Loginov, N. A. Adams, and A. A. Zheltovodov, "Large-eddy simulation of shock-wave/turbulent-boundarylayer interaction," Journal of Fluid Mechanics, vol. 565, pp. 135-169, 2006.
[12] Q. Li and C. Liu, "Numerical investigations on the effects of the declining angle of the trailing-edge of MVG," in Proceedings of the 48th AIAA Aerospace Sciences Meeting, Orlando, Fla, USA, 2010, AIAA 2010-714.

[13] F. Lu, A. Pierce, and Y. Shih, "Experimental study of near wake of micro vortex generators in supersonic flow," AIAA paper 2010-4623.

[14] G. S. Jiang and C. W. Shu, "Efficient implementation of weighted ENO schemes," Journal of Computational Physics, vol. 126, no. 1, pp. 202-228, 1996.

[15] C. Liu and L. Chen, "Study of mechanism of ring-like vortex formation in late flow transition," AIAA Paper 2010-1456. 

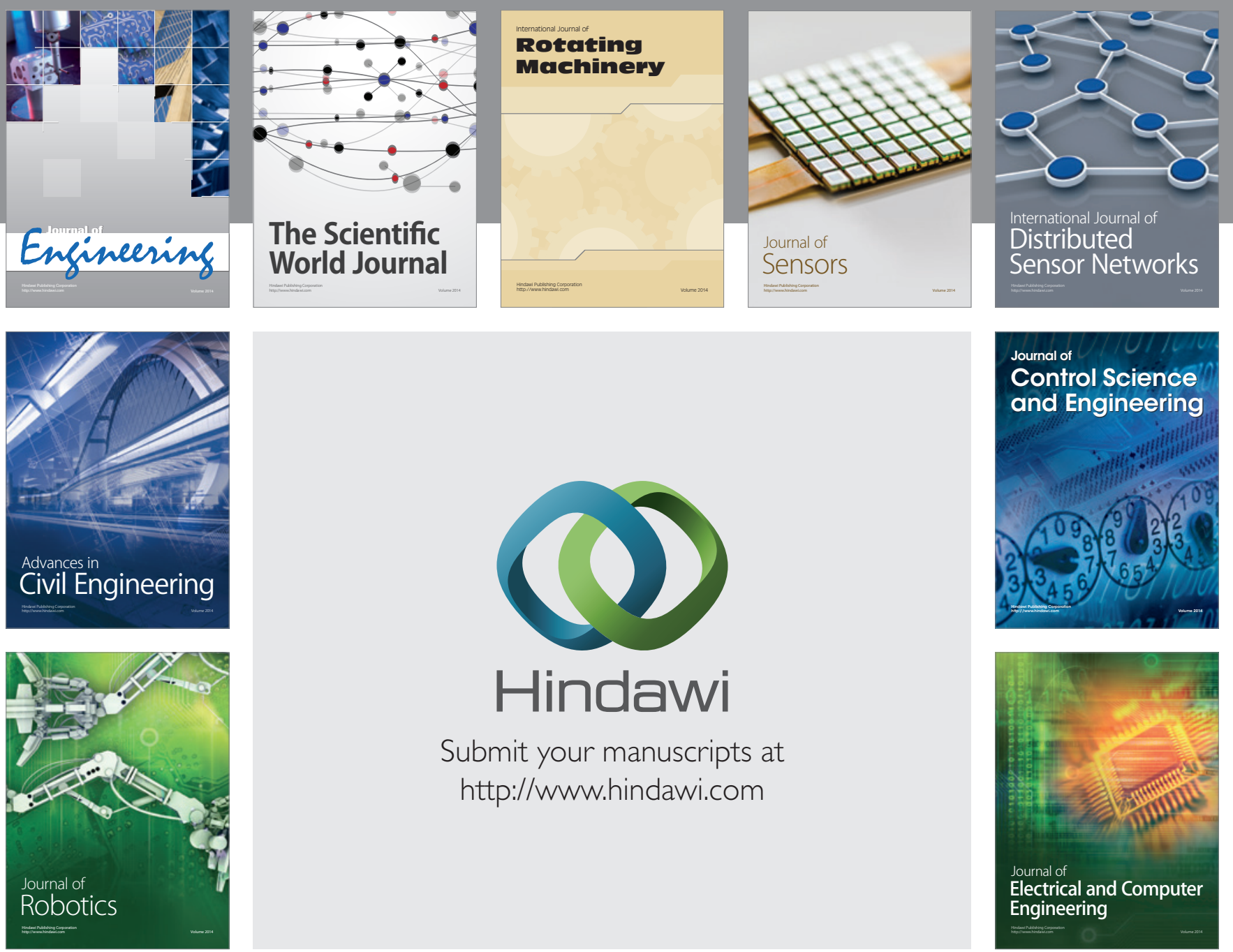

Submit your manuscripts at

http://www.hindawi.com
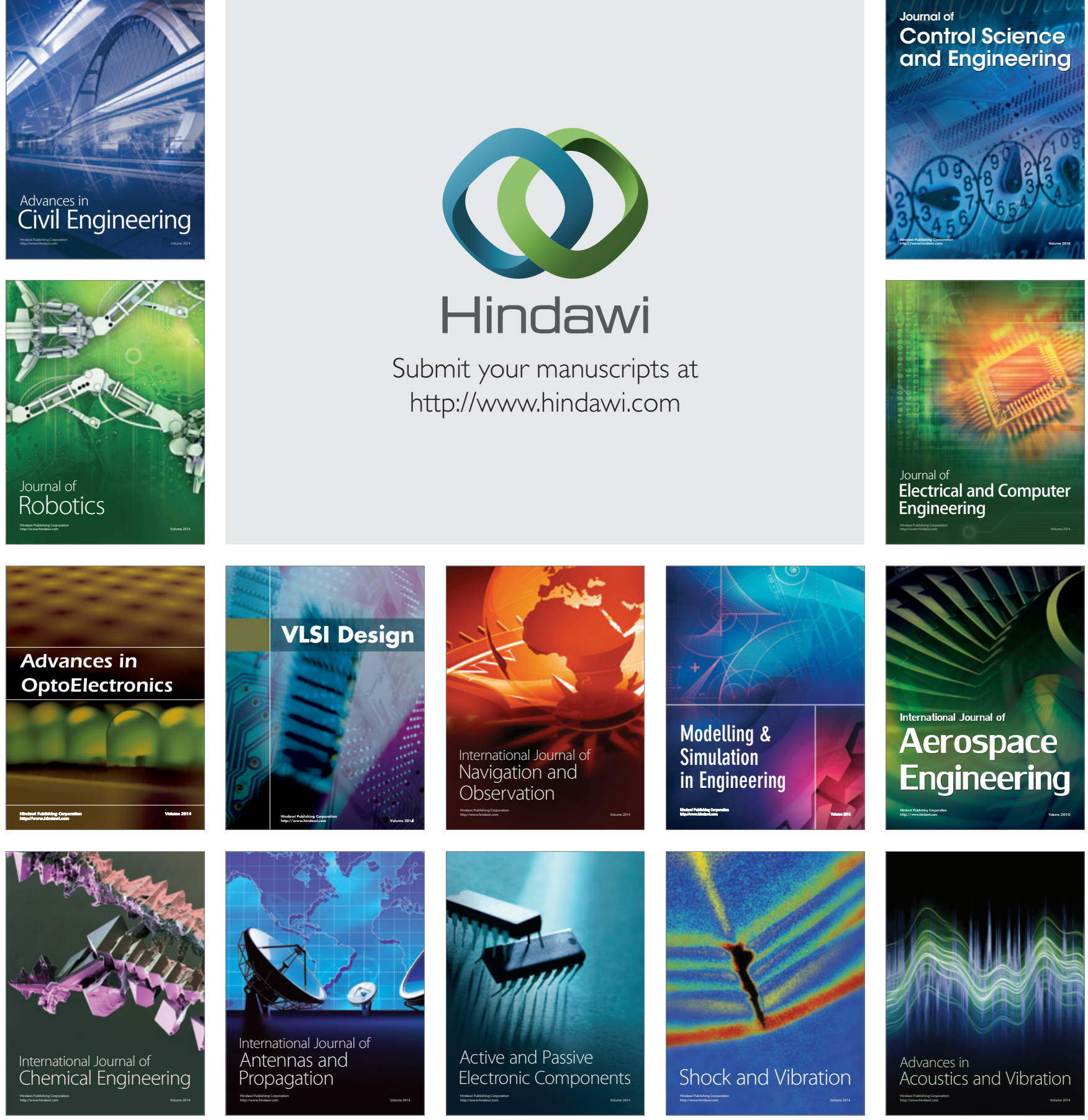